\title{
Análise comparativa da proliferação celular entre carcinomas de células escamosas orais HPV-positivos e HPV-negativos
}

\author{
Comparative analysis of the cellular proliferation between HPV-positive and HPV-negative \\ oral squamous cell carcinomas
}

Danielle Albuquerque Pires Rocha'; Lélia Batista de Souza²; Leão Pereira Pinto³

\begin{abstract}
unitermos
Carcinoma de células

escamosas oral

HPV

Proliferação celular

Ki-67

\section{resumo}

Introdução: O carcinoma de células escamosas oral (OSCC) representa a neoplasia maligna mais freqüente em boca, e, entre os agentes etiológicos implicados, o papilomavírus humano (HPV) tem sido extensivamente estudado nos últimos anos. Objetivo: Analisar comparativamente os índices de proliferação celular em OSCCs HPV-negativos e HPV-positivos. Material e método: A amostra consistiu em 11 casos de OSCCs HPV-positivos (10 infectados por HPV-18 e um por HPV-16 e 18) e 13 HPVnegativos, previamente analisados quanto à presença ou ausência, bem quanto à tipagem viral por proteína $\mathrm{C}$ reativa (PCR) (primers GP5+/GP6+) e hibridização dot blot, respectivamente. No método imunoistoquímico utilizou-se a técnica da estreptoavidina-biotina, com anticorpo para a proteína nuclear Ki-67. Resultados: $\mathrm{O}$ teste estatístico não-paramétrico de Mann-Whitney revelou que não houve diferença estatisticamente significativa entre os grupos HPV-positivo e HPV-negativo $(p=0,72)$. Discussão: Os estudos semelhantes a estes são poucos e não são concordantes em demonstrar maior atividade proliferativa tumoral nos casos HPV-positivos em relação aos HPV-negativos, seja através da análise da expressão de proteínas relacionadas ao ciclo celular, seja na análise direta da fração proliferativa tumoral. Conclusão: Não houve diferença no índice de proliferação celular entre os grupos de OSCCs HPV-positivo e HPV-negativos.
\end{abstract}

Background: Oral Squamous Cell Carcinoma (OSCC) is the most common malignant tumor in the mouth, and the human papillomavirus (HPV) has been hardly studied as a possible etiologic agent. Objectives: The aim of this study was to compare the rates of cell proliferation in HPV-positive and HPV-negative OSCC, using the immunohistochemical antibody Ki-67. Material and method: The sample consisted of 11 cases HPV-positive OSCC (10 cases infected with HPV-18 and 1 case infected with HPV-16 and 18) and 13 cases HPV-negative OSCCS, previously analyzed regarding the presence or absence of HPV, as well as the viral type, using PCR (primers GP5+/GP6+) and dot blot hybridization, respectively. Immunohistochemical study was performed by streptoavidin-biotin technique with antibody against nuclear protein Ki-67. Results: The mean of positivity index of the HPV-positive OSCC (17.7\%) was greater than HPV-negative OSCC (14.2\%), however the statistic analysis showed that standard deviation in both groups was very high, almost equal the mean (14\% and $9.5 \%$, respectively). The Mann-Whitney non-parametric statistic test disclosed that there wasn't a significantly statistical difference between the groups. Discussion: Similar studies to these are few and they are not concordant in demonstrating a bigger tumoral proliferative activity in those cases infected by HPV in relation to those not infected, either through the analysis of the protein expression related to the cellular cycle as in the direct analysis of the tumoral proliferative fraction. Conclusion: There were not differences in the rates of cell proliferation between the HPV-positive and HPV-negative grups.

1. Mestre em Patologia Oral.

2. Doutora em Patologia Oral; professora do Programa de Pós-Graduação em Patologia Oral da Universidade Federal do Rio Grande do Norte (UFRN).

3. Doutor em Patologia Oral; professor do Programa de Pós-graduação em Patologia Oral da UFRN.

Trabalho realizado no Laboratório de Anatomia Patológica do Programa de Pós-graduação em Patologia Oral da UFRN e baseado em dissertação apresentada ao Programa de Pós-Graduação em Patologia Oral da UFRN como parte dos requisitos para obtenção do título de mestre em Patologia Oral. 


\section{Introdução}

O carcinoma de células escamosas oral (OSCC) é a neoplasia maligna mais freqüente em mucosa oral, correspondendo a mais de $90 \%$ dos tumores malignos nesta localização. Em sua etiologia, agentes químicos, físicos e biológicos, além da predisposição genética, têm sido implicados. Na carcinogênese química, o fumo e o álcool representam os fatores mais significativos no desenvolvimento dessa entidade ${ }^{(2,31)}$. Na carcinogênese física, é comprovada a ação deletéria da radiação ultravioleta no DNA celular, clinicamente evidenciado pelo efeito da radiação solar na etiologia do carcinoma epidermóide de lábio inferior ${ }^{(13)}$.

Nas últimas décadas, a participação dos agentes biológicos, especialmente o papilomavírus humano (HPV), na etiologia do OSCC tem sido avaliada, por isso numerosas pesquisas têm sido realizadas na tentativa de se estabelecer uma fiel correlação entre o HPV e esta neoplasia, à semelhança do que acontece com o câncer de colo de

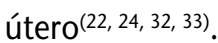

Os HPVs são vírus de DNA, epiteliotrópicos, não-envelopados, com genoma circular de fita dupla e cerca de 8 mil pares de base, medindo $55 \mathrm{~nm}$ de diâmetro. Seu genoma pode ser dividido em três regiões: uma região longa de controle (long control region [LCR]) e as regiões precoce $(\mathrm{E})$ e tardia (L). As regiões $\mathrm{E}$ e $\mathrm{L}$ codificam as proteínas virais, enquanto a LCR é chamada de região não-codificante(23).

A ação oncogênica desse vírus parece estar relacionada a interferências em mecanismos importantes que controlam a proliferação celular. As oncoproteínas virais E6 e E7 são as mais importantes nesse processo, pois são capazes de ligarem-se às proteínas p53 e pRb, respectivamente, e interferirem em suas funções normais ${ }^{(23,26)}$. Além disso, a proteína E6 também apresenta importante papel na ativação da telomerase, contribuindo para o processo de imortalização dos ceratinócitos ${ }^{(11)}$.

Tendo em vista o papel das oncoproteínas virais (principalmente E6 e E7) dos HPVs de alto risco na desregulação do controle da proliferação celular, estudos têm avaliado se lesões cancerizáveis e malignas HPV-positivas exibem níveis de proliferação celular aumentados em relação às HPV-negativas $(3,4,7,17)$.

Um dos métodos mais comuns entre os patologistas para se detectar e quantificar as células em proliferação é a imunoistoquímica, sendo o Ki-67 o anticorpo mais utilizado(10, 27).

O objetivo desta pesquisa foi avaliar e comparar a expressão imunoistoquímica do marcador de proliferação celular Ki-67 entre espécimes de carcinomas epidermóides orais HPV-positivos e HPV-negativos, cujo DNA-HPV foi detectado pela técnica da reação em cadeia da polimerase (PCR) para verificar se há diferença de expressão entre os dois grupos de estudo.

\section{Material e métodos}

\section{Amostra}

Esta pesquisa consistiu em análises quantitativa e comparativa da expressão do Ki-67 entre 11 casos de OSCC HPV-positivos e 13 de OSCC HPV-negativos fixados em formol a $10 \%$ e emblocados em parafina. Foram analisados 24 casos de OSCC provenientes dos arquivos dos laboratórios de anatomia patológica do Hospital Dr. Luiz Antônio (Natal-RN) e do Programa de Pós-Graduação em Patologia Oral da Universidade Federal do Rio Grande do Norte (UFRN). A presença ou ausência do DNA do HPV foi verificada através do método da $P C R$, utilizando-se para isso os primers genéricos GP5+/GP6+, e o tipo viral foi analisado por hibridização dot blot em estudo prévio realizado por Oliveira ${ }^{(14)}$ e Soares ${ }^{(22)}$, sendo o HPV-18 (11 casos) o tipo viral encontrado, com um dos casos apresentando também DNA de HPV-16. Esta pesquisa foi aprovada pelo Comitê de Ética em Pesquisa do Hospital Dr. Luiz Antônio (Natal-RN).

\section{Análise imunoistoquímica}

Os espécimes emblocados em parafina foram submetidos a cortes de $3 \mu \mathrm{m}$ de espessura e montados em lâminas de vidro previamente preparadas com adesivo à base de organosilano (3-aminopropiltrietoxi-silano, Sigma Chemical Co, St. Louis, MO, USA) para realização da técnica imunoistoquímica pelo método da estreptoavidina-biotina-peroxidase. A recuperação antigênica foi realizada em stemear por 30 min, com solução de citrato em pH 6. Aos cortes foi aplicado anticorpo dirigido contra a proteína nuclear Ki-67 (Dako Corporation, clone Ki-5S), com diluição de 1:100, com incubação overnight. A contracoloração foi feita com hematoxilina de Mayer.

Foram consideradas positivas as células cujos núcleos exibiram coloração acastanhada, independente da intensidade da coloração. A análise quantitativa da expressão da proteína Ki-67 foi realizada utilizando-se um índice de positividade (IP). Em aumento de 100x foi selecionada a área de maior expressão do Ki-67 no espécime e, com aumento final de $1.000 x$, foram contados os núcleos positivos 
e negativos. O IP foi então calculado através da relação do número de células Ki-67 positivas por 500 células contadas em cada caso estudado, e os valores foram expressos em porcentagem (adaptado de Piattelli et al., 2002) ${ }^{(15)}$.

\section{Análise estatística}

Para verificar a distribuição dos dados utilizou-se o teste de Kolmogorov Smirnov (teste de normalidade), e para comparar os grupos estudados optou-se pela realização do teste não-paramétrico de Mann-Whitney (com nível de significância de 5\%).

\section{Resultados}

A marcação imunoistoquímica do Ki-67 foi intensa na maioria dos casos, exibindo coloração acastanhada observável e distinguível dos núcleos não marcados, tanto nos casos HPV-positivos quanto nos HPV-negativos (Figuras 1 e 2). Observou-se marcação em células das camadas basal e suprabasal, em células localizadas nos centros dos ninhos e lençóis, bem como, embora em menor proporção, em células superficiais, além de evidente marcação em células que estavam sofrendo mitose.

A média dos índices de positividade (IP) dos OSCCs HPVpositivos foi um pouco maior $(17,7 \%)$ do que a dos OSCCs HPV-negativos (14,2\%). Esses índices variaram bastante em ambos os grupos, principalmente no grupo HPV-positivo; tanto o menor IP $(0,8 \%)$ quanto o maior $(42,8 \%)$ eram de casos pertencentes a este grupo. No grupo HPV-negativo o menor índice de positividade foi $2 \%$ e o maior, $32,7 \%$ (Tabelas 1 e 2). Analisando as medianas dos grupos, observamos que estiveram muito próximas (HPV-positivo = 18,6 e HPV-negativo = 15,6), como demonstrado na

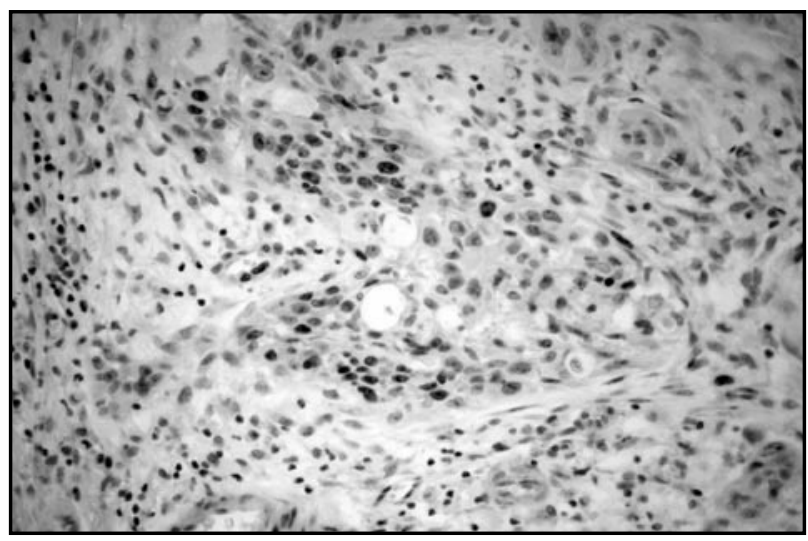

Figura 1 - Marcação imunoistoquímica pelo Ki-67 em carcinoma de células escamosas oral HPV-positivo, evidenciando células em proliferação no centro e na periferia dos ninhos tumorais

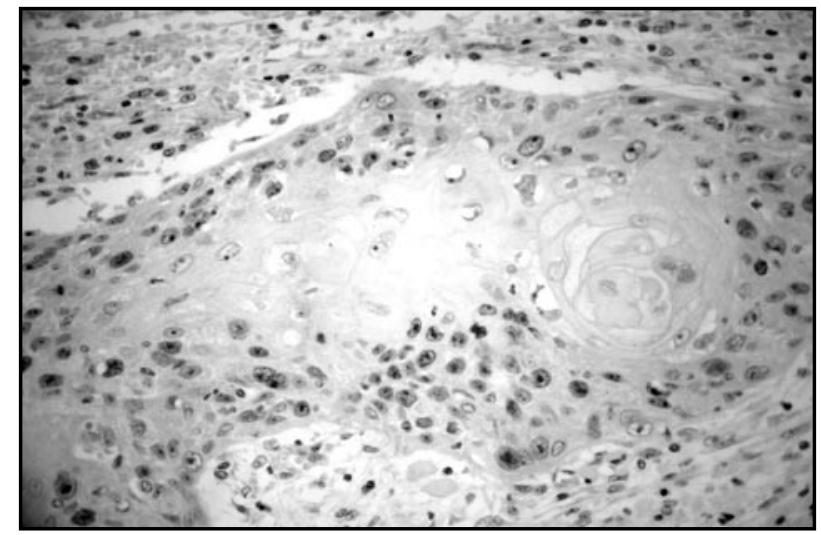

Figura 2 - Marcação imunoistoquímica pelo Ki-67 em carcinoma de células escamosas oral HPV-negativo, evidenciando um alto índice de células em proliferação

\begin{tabular}{|c|c|}
\hline Tabela 1 & $\begin{array}{l}\text { Dados relativos aos índices de } \\
\text { positividade dos casos de OSCC HPV- } \\
\text { positivos (Natal, RN - 2006) }\end{array}$ \\
\hline Caso & $\mathbb{I P}(\%)$ \\
\hline 1 & 12,8 \\
\hline 2 & 0,8 \\
\hline 3 & 21,8 \\
\hline 4 & 18,6 \\
\hline 5 & 4,4 \\
\hline 6 & 1,8 \\
\hline 7 & 26 \\
\hline 8 & 31 \\
\hline 9 & * \\
\hline 10 & 42,8 \\
\hline 11 & * \\
\hline Média (DP) & $17,7( \pm 14,3)$ \\
\hline
\end{tabular}

*Casos eliminados das análises quantitativa e estatística. IP: índice de positividade; OSCC: carcinoma de células escamosas oral; DP: desvio- padrão.

\begin{tabular}{lc}
\cline { 2 - 2 } Tabela 2 & $\begin{array}{c}\text { Dados relativos aos índices de } \\
\text { positividade dos casos de OSCC } \\
\text { HPV-negativos (Natal, RN - 2006) }\end{array}$ \\
\hline Caso & IP (\%) \\
\hline 1 & 8,22 \\
2 & 18,6 \\
3 & 18,2 \\
4 & 3,4 \\
5 & 19,9 \\
6 & $*$ \\
\hline
\end{tabular}


7

8

9

10

11

12

13

Média (DP)

*Caso eliminado das análises quantitativa e estatística.

IP: índice de positividade; OSCC: carcinoma de células escamosas oral; DP: desvio-padrão.

Figura 3. O teste de Kolmogorov Smirnov revelou que os dados apresentavam uma distribuição normal $(0,16 \mathrm{e}$ $0,17)$. Porém, devido à grande variação dos dados, com o desvio padrão muito próximo da média (14,3 para o grupo HPV-positivo e 9,5 para o grupo HPV-negativo), optou-se por utilizar o teste não-paramétrico de Mann-Whitney, cujo resultado indicou não haver diferença estatisticamente significativa entre os dois grupos $(p=0,72)$.

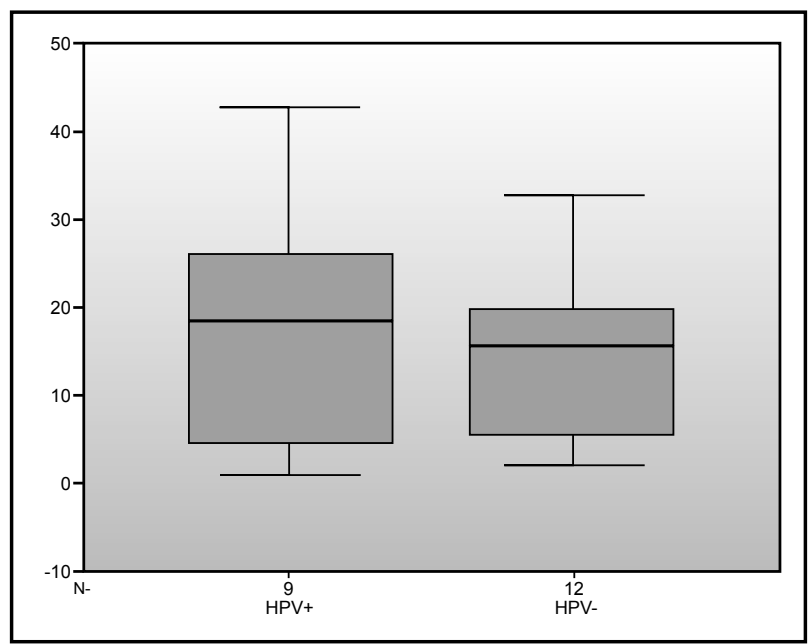

Figura 3 - Box blot indicativo da análise não-paramétrica dos índices de positividade de marcação pelo Ki-67 nos grupos HPV-positivos e HPV-negativos

\section{Discussão}

Nos últimos anos, a participação do HPV no câncer de boca tem sido extensivamente estudada por vários

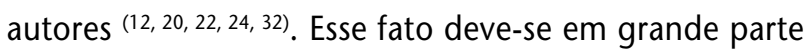
ao aumento do diagnóstico das doenças sexualmente transmissíveis presentes na cavidade oral e à associação de alguns tipos de HPV oncogênicos com malignidades anogenitais ${ }^{(11,16)}$, particularmente com o carcinoma de células escamosas de colo de útero, cuja mucosa exibe semelhanças com o epitélio de mucosa oral ${ }^{(30)}$.
Algumas das proteínas sintetizadas por este vírus têm a capacidade de interferir nos mecanismos que controlam a proliferação da célula hospedeira que estão infectando, principalmente interferindo no funcionamento normal das proteínas sintetizadas pelos antioncogenes p53 e $\mathrm{pRb}^{(34)}$.

Considerando-se essa capacidade que o HPV apresenta em induzir a proliferação celular, já evidenciada nas lesões benignas também causadas por este vírus, o objetivo desta pesquisa foi analisar comparativamente os índices de proliferação celular em OSCCs HPV-positivos e HPVnegativos, com o auxílio do marcador imunoistoquímico de proliferação celular Ki-67.

Escolheu-se o marcador Ki-67 porque, segundo a literatura, várias de suas propriedades o fazem um marcador de proliferação celular bastante confiável. Entre essas propriedades, destaca-se o Ki-67, que está presente em todas as fases do ciclo celular, exceto na fase $\mathrm{G} 0$, tendo meia-vida curta (em torno de 1 hora) e sendo rapidamente metabolizado no final da mitose. Portanto, não há expressão do Ki-67 em células que já tenham deixado o ciclo. Essa proteína não está envolvida no sistema de reparação do DNA, e sua expressão restringe-se aos compartimentos proliferativos dos tecidos. Adicionalmente, o Ki-67 não sofre influências marcantes da fixação e do processamento histológicos ou da diluição do anticorpo ${ }^{(6,10,19,28,29)}$.

Nossos resultados demonstraram que não houve diferença estatisticamente significativa entre os dois grupos analisados $(p=0,72)$, fato este também verificado por Donofrio et $a l .^{(3)}$, que não encontraram correlação entre presença de HPV e índices aumentados de proliferação celular em lesões cancerizáveis (displasias leves, moderadas e severas) e OSCCs microinvasivos. Entretanto, esses autores utilizaram um método menos sensível, a hibridização in situ (HIS), para detecção do HPV do que a PCR utilizada por nós. Além disso, os autores usaram o anticorpo imunoistoquímico antígeno nuclear de proliferação celular (PCNA), enquanto nós utilizamos o Ki-67, que, segundo a literatura, é superior àquele ${ }^{(9,10,28)}$.

Utilizando uma metodologia mais semelhante à do presente estudo, Kojima et al. ${ }^{(7)}$, diferentemente dos nossos resultados, encontraram um progressivo e significativo aumento dos índices de proliferação celular entre mucosa oral normal, OSCC HPV-38-negativo e OSCC HPV-38positivo, empregando PCR e PCNA. El-Mofty e Lu ${ }^{(4)}$ também encontraram escores aumentados de marcação pelo Ki-67 em carcinomas de células escamosas de cabeça e pescoço HPV-positivos em relação aos HPV-negativos. 
Os estudos que analisam a expressão de algumas das principais proteínas reguladoras do ciclo celular em carcinomas escamosos infectados e não-infectados por HPV não são concordes quanto a verificarem diferenças estatisticamente significativas entre os grupos. Em OSCC, Oliveira ${ }^{(10)}$ não encontrou diferença na expressão da p53 entre os grupos; Soares ${ }^{(22)}$ não verificou diferença na expressão da p21, mas, quanto à pRb, verificou níveis bastante aumentados nas lesões infectadas, sugerindo acúmulo da $\mathrm{pRb}$, que estaria inativa por estar ligada à proteína $\mathrm{E} 7$ dos HPVs; Azzimonti et al.(1), por sua vez, estudando carcinomas escamosos de laringe e orofaringe, não encontraram diferenças na expressão da pRb entre lesões HPV-positivas e HPV-negativas.

Pode-se inferir que, em cavidade oral, não há consenso sobre o fato de a proliferação celular estar aumentada nos OSCCs infectados por HPV. Entendemos que isso se deva à escassez de estudos semelhantes, bem como às diferentes metodologias empregadas nestes, seja para detecção do HPV, seja na escolha do anticorpo imunoistoquímico para detecção e quantificação das células proliferantes, conforme citam alguns autores $(3,7,17)$.

Acreditamos que apenas a detecção do DNA do HPV nos casos de OSCC não é evidência suficiente para o estabelecimento de uma relação causal, pois altos índices desse vírus, incluindo os tipos oncogênicos 16 e 18, também são encontrados em mucosa oral normal, inclusive de crianças e adolescentes ${ }^{(8,25)}$. É necessário que essa presença viral esteja acompanhada de achados condizentes com o mecanismo de ação oncogênica do vírus. Quanto à indução da proliferação celular, não foi verificada neste estudo.

A importância do estabelecimento dessa relação reside também na busca de parâmetros para estabelecimento de prognóstico. Alguns estudos têm sido realizados com a finalidade de se detectar e quantificar as células tumorais em proliferação como um parâmetro importante para o prognóstico do paciente, bem como correlacioná-la com algumas características clinicopatológicas, como estágio TNM (T: tumor, $\mathrm{N}$ : linfonodo, M: metástase), grau histológico de malignidade, recidiva tumoral e sobrevida do paciente ${ }^{(5,10,18,29)}$.

Porém, o real valor clínico dessa possível indicação de agressividade tumoral é ainda objeto de debate. É discutível, por exemplo, quando consideramos os protocolos terapêuticos dos diversos centros de tratamento, pois a resposta à radioterapia, por exemplo, é mais eficaz em tumores que exibem maiores índices de proliferação, o que não é verdadeiro para a cirurgia e é variável para a quimioterapia ${ }^{(6,19)}$.

A real participação do HPV na etiologia do OSCC é, até agora, inconclusiva. Suas propriedades transformantes relacionadas à indução da proliferação celular nas lesões sediadas neste sítio anatômico não são observadas consistentemente na literatura, seja através da análise da expressão das proteínas reguladoras do ciclo, seja pelo estudo direto da fração proliferativa tumoral, o que reforça a necessidade de que mais estudos nessa área do conhecimento sejam realizados na busca de um melhor entendimento do papel biológico do HPV nessa lesão.

\section{Referências}

1. AZZIMONTI, B. et al. Altered patterns of the interferoninducible gene IFI16 expression in head and neck squamous cell carcinoma: immunohistochemical study including correlation with retinoblastoma protein, human papilomavírus infection and proliferation index. Histopathol, v. 45, n. 6, p. 560-72, 2004.

2. BUNDGAARD, T.; BENTZEN, S. M.; SEGAARD, H. Histological differentiation of oral squamous cell cancer in relation to tobacco smoking. Oral Oncol Eur J Cancer, v. 31B, n. 2, p. 118-21, 1995.

3. DONOFRIO, V. et al. Prognostic evaluation of HPVassociated precancerous and microinvasive carcinoma of the oral cavity: combined use of nucleolar organiser regions (AgNOR) and proliferating cell nuclear antigen (PCNA). Oral Oncol Eur J Cancer, v. 31B, n. 3, p. 174-80, 1995

4. EL-MOFTY, S. K.; LU, D. W. Prevalence of Human
Papillomavirus type 16 DNA in squamous cell carcinoma of the palatine tonsil, and not the oral cavity, in young patients. A distinct clinicopathologic and molecular disease entity. Am J Surg Pathol, v. 27, n. 11, p. 1463-70, 2003.

5. FUMIC-DUNKIC, L. et al. Retrospective analysis of Ki-67 antigen expression in paraffin tissue blocks of laryngeal squamous cell carcinoma. Am J Otolaryngol, v. 24, n. 2, p. 106-10, 2003.

6. GIROD, S. C. et al. Proliferative activity and loss of function of tumor suppressor genes as 'biomarkers' in diagnosis and prognosis of benign and preneoplastic oral lesions and oral squamous cell carcinoma. Br J Oral Maxillofac Surg, v. 36, n. 4, p. 252-60, 1998.

7. KOJIMA, A. et al. Human papillomavirus type 38 infection in oral squamous cell carcinomas. Oral Oncol, v. 38, n. 6, p. 591-6, 2002. 
8. KOJIMA, A. et al. Human papillomaviruses in the normal oral cavity of children in Japan. Oral Oncol, v. 39, n. 8, p. 821-8, 2003.

9. KRECICKI, T. et al. Epidermal growth factor receptor (EGFR), proliferating cell nuclear antigen (PCNA) and Ki-67 antigen in laryngeal epithelial lesions. Oral Oncol, v. 35, n. 2, p. 180-6, 1999.

10. KUROKAWA, H. et al. The relationship of the histologic grade et the deep invasive front and the expression of Ki-67 antigen and p53 protein in oral squamous cell carcinoma. J Oral Pathol Med, v. 34, n. 10, p. 602-7, 2005.

11. MCMURRAY, H. R. etal. Biology of human papillomaviruses. Int J Exp Path, v. 82, n. 1, p. 15-33, 2001.

12) MILLER, C. S.; JOHNSTONE, B. M. Human papillomavirus as a risk factor for oral squamous cell carcinoma: a meta-analysis, 1982-1997. Oral Surg Oral Med Oral Pathol Oral Radiol Endod, v. 91, n. 6, p. 622-35, 2001.

13. NEVILLE, B. W. et al. Patologia epitelial. In: NEVILLE, B. W. Patologia oral \& maxilofacial. Rio de Janeiro: Guanabara Koogan, 2004. p. 303-72, cap. 10.

14. OLIVEIRA, M. C. Papilomavírus humano (HPV) de alto risco e sua relação com a expressão das proteínas p53 e bcl-2 em carcinoma epidermóide oral. 2005. 105p. Tese (Doutorado em Patologia Oral) - Departamento de Odontologia da Universidade Federal do Rio Grande do Norte, Natal.

15. PiATtELLI, A. et al. Prevalence of p53, bcl-2, and ki67 immunoreactivity and of apoptosis in normal oral epituhelium and in premalignant and malignant lesions of the oral cavity. J Oral Maxillofac Surg, v. 60, n. 5, p. 532-40, 2002

16. SCULLY, C. Oral squamous cell carcinoma; from an hypothesis about a virus, to concern about possible sexual transmission. Oral Oncol, v. 38, n. 3, p. 227-34, 2002.

17. SHINDOH, M. et al. Detection of Human Papillomavirus DNA sequences in oral squamous cell carcinomas and their relation to p53 and proliferating cell nuclear antigen expression. Cancer, v. 76, n. 9, p. 1513-21, 1995.

18. SILVA, S. D. et al. Expression of fatty acid synthase, ErbB2 and $\mathrm{Ki}-67$ in head and neck squamous cell carcinoma. A clinicopathological study. Oral Oncol, v. 40, n. 7, p. 688-96, 2004.

19. SITTEL, C. et al. Prognostic significance of Ki-67 (MIB), PCNA and p53 in cancer of the oropharynx and oral cavity. Oral Oncol, v. 35, n. 6, p. 583-9, 1999.

20. SOARES, C. P. Papilomavírus humano (HPV): um estudo de revisão. Rev Cienc Farm, v. 20, n. 1, p. 11-34, 1999.

21. SOARES, C. P. et al. Presença do papilomavírus humano em lesões malignas de mucosa oral. Rev Soc Bra Med Trop, v. 35, n. 5, p. 439-44, 2002.

22. SOARES, R. C. Estudo da detecção do DNA do PHV e da expressão imuno-histoquímica de proteínas do ciclo celular no carcinoma epidermóide oral. 2005. 95p. Tese (Doutorado em Patologia Oral) - Departamento de Odontologia da Universidade Federal do Rio Grande do Norte, Natal.

23. SUGERMAN, P. B.; SHILLITOE, E. J. The high risk human papillomaviruses and oral câncer; evidence for and against a causal relationship. Oral Dis, v. 3, n. 3, p. 130-47, 1997.

24. SUGIYAMA, M. et al. Detection of human papillomavirus16 and HPV-18 in normal, dysplastic and malingnant oral epithelium. Oral Surg Oral Med Oral Pathol Oral Radiol Edond, v. 95, n. 5, p. 594-600, 2003.

25. TERAI, M. etal. High prevalence of human papillomaviruses in the normal oral cavity of adults. Oral Microbiol Immunol, v. 14, n. 4, p. 201-5, 1999.

26. TERAI, M.; TAKAGI, M. Human papillomavirus in the oral cavity. Oral Med Pathol, v. 6, p. 1-12, 2001.

27. TUMULURI, V.; THOMAS, G. A.; FRASER, I. S. Analysis of the Ki-67 antigen et the invasive tumour front of human oral squamous cell carcinoma. J Oral Pathol Med, v. 31, n. 10, p. 598-604, 2002.

28. VAN DIEST, P.J.; BRUGAL, G.; BAAK, J. P. A. Proliferation markers in tumours: interpretation and clinic value. $J$ Clin Pathol, v. 51, n. 10, p. 716-24, 1998.

29. VICENT, J. C. et al. Expression of ciclin D1 and ki67 in squamous cell carcinoma of the oral cavity: clinicopathological and prognostic significance. Oral Oncol, v. 38, n. 3, p. 301-8, 2002.

30. VILLA, L. L. Human papillomaviruses and cervical cancer. Advanc Canc Res, v. 71, p. 321-41, 1997.

31. WIGHT, A. J.; OGDEN, G. R. Possible mechanisms by which alcohol may influence the development of oral cancer: a review. Oral Oncol, v. 34, n. 6, p. 441-7, 1998.

32. ZHANG Z.-Y. et al. Human papillomavirus type 16 and 18 in oral squamous cell carcinoma and normal mucosa. Oral Maxillofac Surg, v. 33, p. 71-4, 2004.

33. Zur HAUSEN, H. Papillomavirus infection: a major cause of human cancers. Bioch Biophy Acta, v. 1288, n. 2, p. F55-F78, 1996.

34. Zur HAUSEN, H. Papillomaviruses causing cancer: easion from host-cell control in early events in carcinogenesis. J Natl Cancer Inst, v. 92, n. 9, p. 690-8, 2000. 